\title{
TRANSPORT PROPERTIES IN 2,6-DIAMINO ANTHRAQUINONE
}

\author{
S.M. KHALIL \\ Physics Department, Alexandria University, Alexandria, Egypt \\ AND S. DARWISII \\ Physics Department, El-Minia University, El-Minia, Egypt \\ (Received August 3, 1993; revised version December 14, 1993)
}

\begin{abstract}
Current density-voltage characteristics were obtained from 2,6-diamino anthraquinone samples using ohmic aluminium electrodes. Results showed that at low voltage the conduction process was olımic, whilc at high voltage space-charge-limited conduction controlled by a single dominant trap level was presented. Thickness dependence measurements proved that the trapping sites were located at a discrete cnergy level. The transition voltage, $V_{l}$, between ohmic and space-charge-limited conduction was approximately proportional to the square of the sample thickness and was found to be temperature independent. The temperature dependence of ohmic and space-charge-limited current densities have been investigated. The results were interpreted in terms of extrinsic nature of olmmic conduction. Traps with density $\approx 2 \times 10^{24} \mathrm{~m}^{-3}$ localed at $0.50 \pm 0.03 \mathrm{eV}$ below the conduction band edge have becn observed.
\end{abstract}

PACS numbers: 72.20.-i

\section{Introduction}

The study of thie temperature dependence of ohmic and space-charge-limited (SCL) currents in scmiconductors and insulators is capable of providing considerable insight into the mechanism of charge transport and carrier trapping in these materials. The group of organic compounds known as antliraquinone is convenient for such a study. Anthraquinone $(A Q)$ and its derivatives are a class of organic compounds which fall under the group of disperse dyes [1]. These compounds are of interest because they can promote photo-tendering of textiles. AQs derivatives are widely used as commercial dyes for both natural and synthetic fibers. The dark and photoconduction of $\Lambda$ Qs have been investigated [1-4] trying to explain the origin of the observed conductivity of such compounds in terms different modes of carrier generation. 
In this paper we report measurements of ohmic and SCL currents in 2,6-diamino anthraquinone (2,6-DAAQ), with charge carriers injectcd from the electrodes and generated in the bulk towards understanding of the conduction mechanism in the 2,6-DAAQ compound.

\section{Experimental details}

The 2,6-DAAQ used in these measurements was purchased from Alderich Chemical Company Inc. (USA) and was purified by vacuum sublimation. The original powdered material was compressed in a dic into pellets of 1-4 mm thickness and which were fitted with evaporated film electrodes of aluminium to serve as olımic contacts for electrons to this materials [1]. Silver paste contacts with the electrodes have been used for studying the clectrical measurements. The specimens were mounted onto an electrically heated copper disc, the temperature of which could be held constant to within $1 \mathrm{~K}$ over the rangc of $300 \mathrm{~K}$ to $373 \mathrm{~K}$. The temperaturcs were measured by means of chromel/alumel thermocouple mounted in close proximity to the specimen of interest. The current flowing through a specimen was determined using a conventional de technique and Keithley 610 electrometcr. When the voltage was applied, the currents were allowed to increase to a steady value within few seconds. For consistency the currents were allowed to stabilize for 2 minutes before a reading was taken. All samples used were measured by means of this technique.

\section{Results and discussion}

The Seebeck effect in 2,6-DAAQ was investigated by measuring the electromotive force $\Delta V$ which developed across the specimens when a temperature difference $\Delta T$ was established across them. The experiment was carried out to establish the sign of the majority of carriers. Results varied slightly from a specimen to a specimen, but invariant electrons were observed to be the majority of carriers over the entire temperature range of $300-375 \mathrm{~K}$. The smooth curve of $Q=\Delta V / \Delta T$ versus $T$ is shown in Fig. 1. Recently Narasimharaglavan et al. [1], from their photoconduction studies, have shown that the $\Lambda Q$ s are $n$-type semiconductors with electrons being the majority of charge carriers. Figure 2 shows a typical set of current density-voltage $(J-V)$ characteristics appropriate to the temperature indicated. The curves are characterized by two different slopes, which are approximately 1.0 and 1.9 for the low voltage and high voltage, respectively. IIence 2,6-diamino anthraquinone, in common with other organic materials, exhibits olmic conduction at low applied voltage and space-charge-limited (SCL) conduction governed by a discrete traps at high voltage [5]. The ohmic behaviour in the low voltage region is due to the ncgligible injection of carriers from the contact and the initial current is governed by the thermally excited free carriers. Ilence, within the ohmic region, the current density $J_{\Omega}$ is given by [6]

$$
J_{\Omega}=n e \mu V / d \text {, }
$$

where $n$ is the concentration of thermally generated electrons, $e$ - the electronic charge, $\mu$ - the mobility, $d-$ the sample thickness and $V$ - the dc applied voltage. 


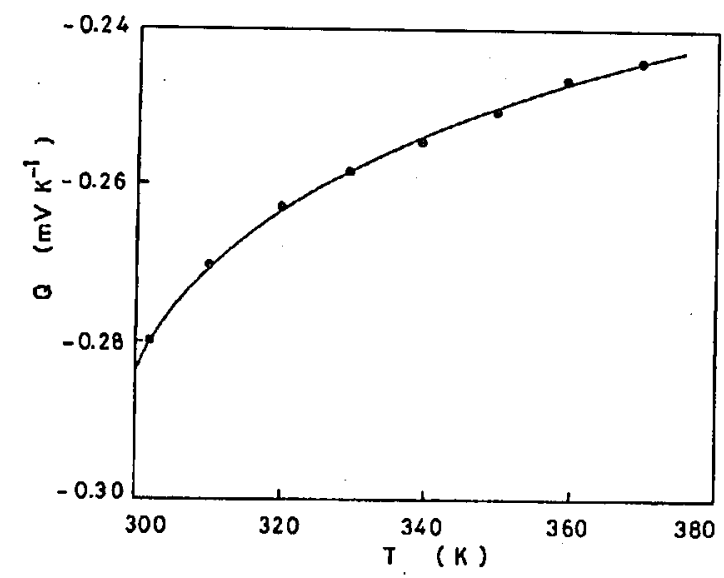

Fig. 1. Temperature depcndence of the Sccbeck coefficient for $n$-type 2,6-DAAQ.

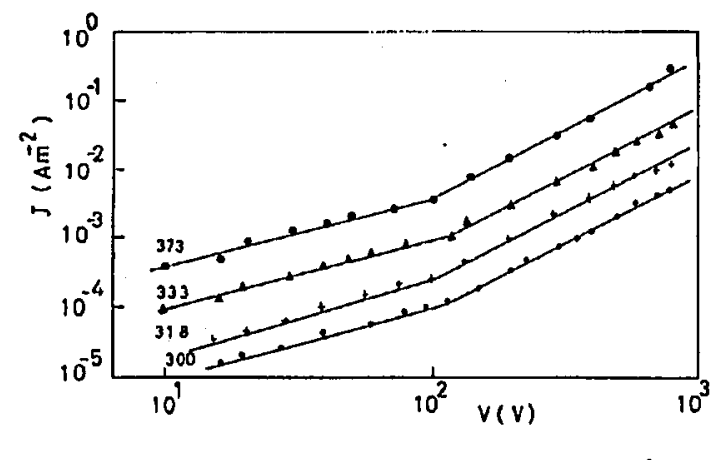

Fig. 2. Current density-voltage curves of different temperatures. Temperatures are given in $\mathrm{K}$.

Within the SCL conduction region, the discrete trap distribution results in a current density, $J_{\mathrm{SCL}}$, an expression given by [5]

$$
J_{\mathrm{SCL}}=\frac{9}{8} \Theta \varepsilon \mu \frac{V^{2}}{d^{3}},
$$

where in addition to the previously defined symbols, $\varepsilon$ is the permittivity and the trapping factor $\Theta$ is the fraction of the total number of charge carriers which are free.

Following the theory of Lampert [5], Eq. (2) is valid for the injection of one type of carrier only in the presence of a single discrete trapping level. Both $J_{\Omega}$ and $J_{\mathrm{SCL}}$ are thermally activated, their activaction energies being contained in $n$ and $\Theta$, respectively. According to Eqs. (1) and (2) the $V_{t}$ voltage, at which the current passing through a specimen converts from being ohmic to SCL, can be defined as:

$$
V_{\mathrm{t}}=\frac{8}{9} \frac{e n d^{2}}{\varepsilon \Theta} \text {. }
$$


Clearly, $V_{\mathrm{t}}$ has a thermal activation cnergy equal to the difference between activation energies for ohmic and SCL conduction. Thus, $V_{t}$ is tempcrature dependent unless the two activation energies are identical.

It can be seen from Fig. 2 that the voltage $V_{t}=(210 \pm 10) \mathrm{V}$, at which the transition from olımic to SCL behaviour takes place, is independent of the temperature, it implics that the sample is extrinsic [7].

For $n$-type material containing $N_{\mathrm{d}}$ donors and $N_{\mathrm{a}}$ acceptors per unit volume (a partly compensated specimen), the density of free electrons responsible for ohmic conduction is given by [7]

$$
n=\frac{\left(N_{\mathrm{d}}-N_{\mathrm{a}}\right)}{N_{\mathrm{t}}} N_{\mathrm{c}} \exp \left(-E_{\mathrm{t}} / k \cdot T\right),
$$

where $N_{\mathrm{c}}$ is the effective density of states in the conduction band, $E_{\mathrm{t}}$ - the energy below the conduction band edge at which the traps are located with the state density $N_{\mathrm{t}}, k-$ Boltzman's constant and $T$ - the absolute temperature.

The electron trapping factor, $\Theta$, for SCL conduction is $[8,9]$

$$
\Theta=\frac{N_{\mathrm{c}}}{N_{\mathrm{t}}} \exp \left(-E_{\mathrm{t}} / k T\right) \text {. }
$$

The measurements of the sample capacitance of a known thickness yielded a permittivity of $\approx 2.05 \times 10^{-11} \mathrm{~F} \mathrm{~m}^{-1}$ (or a relative permittivity of $\approx 2.30$ ) and this value was used in all the following calculations. These measurements were performed at $100 \mathrm{KIIz}$ using Tesla BM 507 impedance meter. Using the set of Eqs. (3), (4) and (5), the donor excess was found to be $\left(N_{\mathrm{d}}-N_{\mathrm{a}}\right) \approx 7 \times 10^{15} \mathrm{~m}^{-3}$. The dependence of $V_{\mathrm{t}}$ on $d$ as predicted by Eq. (3) allows further verification of the model. In Fig. 3 the dependece of $V_{\mathrm{t}}$ on $d$ is shown. The slope of this plot is $: \approx 1.9$ which is in good agreement with the expected linear dependence of $V_{\mathrm{t}}$ on $d^{2}$ from Eq. (3).
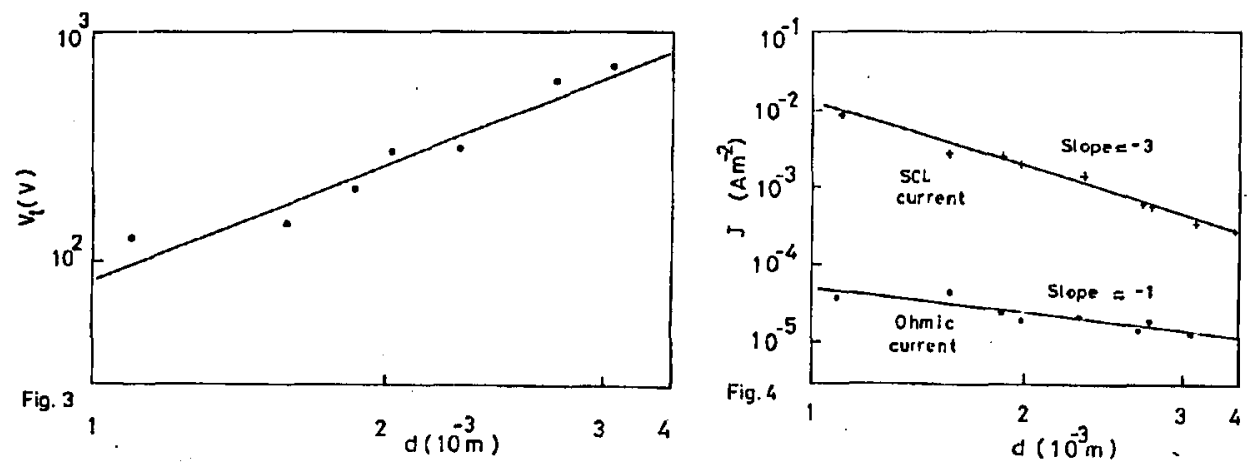

Fig. 3. Dependence of transition voltage $V_{t}$ on thickness $d$.

Fig. 4. Current densities vs. thickness sample for ohmic and SCL conduction. (Temperature $300 \mathrm{~K}$. Applied voltage $25 \mathrm{~V}$ and $500 \mathrm{~V}$ for ohmic and SCL currents, respectively).

A distingushing cliaracteristic of single-carricr SCL current by a single trapping level is the thickness dependence given by Eqs. (2) and (5). In Fig. 4 the 
dependence of current density versus sample thickness for both olımic and SCL regions is shown. It is evident that ohmic conduction and one carrier SCL currents dominated by a single set of traps are observed in our material. More information about material is obtained from the temperature dependence of the SCL and ohmic current densities. The applied voltage of $600 \mathrm{~V}$ and $40 \mathrm{~V}$ were chosen so that the data for both SCL and ohmic regions could be conveniently shown on the same figure. For all the samples studied, the thermal activation energies for ohmic and SCL conduction are identical within an experimental error. Following Schmidlin and Roberts [10], the data shown in Fig. 5 is associated with extrinsic behaviour and a discrete set of traps level, $E_{\mathrm{t}}$, located at $(0.50 \pm 0.03) \mathrm{eV}$ bclow the conduction band edge. Good straight lines are obtained, thus indicating that lattice scattering is dominant. Terefore, the product of mobility and the state density is relatively weakly dependent on temperature in agrcement with the prediction of the band model.

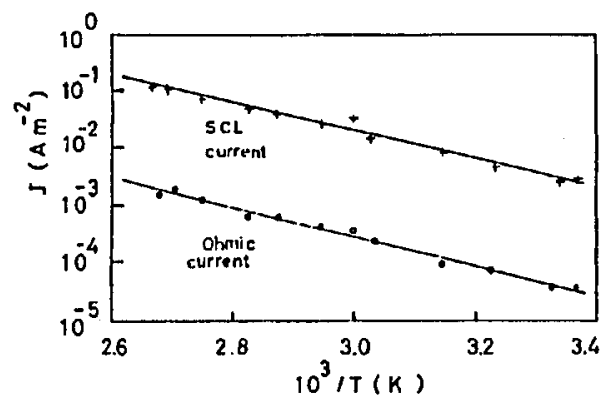

Fig. 5. Variation of current density with reciprocal temperature for olımic and SCL currents.

The intercept on the $\log J$ axis for SCL conduction is given by $\log \left(\mu N_{\mathrm{c}} / N_{\mathrm{t}}\right)$ and immediately yields the trap density, $N_{\mathrm{t}}$, provided that $\mu$ and $N_{\mathrm{c}}$ are known. Assuming the effective density of states in the conduction band to be of the order of the density of molecules (approximately $10^{27} \mathrm{~m}^{-3}$ ) and using electron mobility to be $\approx 1 \times 10^{-4} \mathrm{~m}^{2} \mathrm{~V}^{-1} \mathrm{~s}^{-1}$, then the value of $N_{\mathrm{t}}$ was found to be $2 \times 10^{24} \mathrm{~m}^{-3}$. The nature of traps in 2,6-DAAQ is not clear at the moment, however structural imperfection is a very likely candidate.

\section{Summarizing remarks and conclusions}

Current density-voltage measurements showed ohmic and space-charge-limited conduction at lower and higher voltage levels, respectively. The slopes of the space-charge-limited $\log J$ versus $\log V$ characteristics wcre approximately 1.9 ; while those of $\log J$ versus $\log d$ characteristics were approximately -3 . These results are entirely compatible. The transition voltage, $V_{t}$, between the ohmic and space-charge-limited conduction was approximately proportional to the square of the sample thickness, also in accordance with the theory. The thermal activation 
energies for the ohmic and space-charge-limited regions were approximately identical, it implies that a sample is extrinsic. The value for each region has been found to be $\approx(0.50 \pm 0.03) \mathrm{eV}$, which suggests a trap located at this energy distance from the conduction band edge.

\section{Acknowledgment}

The authors would like to thank Prof. Dr T.G. Abdel-Malik, Department of Physics, Faculty of Science, Minia University (Egypt) for his help and advice through this work.

\section{References}

[1] P.K. Narasimharaghavan, T.S. Varadarajan, IIari Om Yadav, J. Mater. Sci. 28, 337 (1993).

[2] P.K. Narasimharaghiavan, ITari Om Yadav, Shankar, T.S. Varadarajan, J. Mater. Sci., Mater. in Elect. 2, 194 (1991).

[3] V.V. Bhujle, M.R. Padhye, Ind. J. Chem. 9; 1405 (1971).

[4] P. Bentley, J.F. McKeller, G.O. Philips, J. Chem. Soc. Perkin. Trans. 11, 523 (1974).

[5] M.A. Lampert, Rep. Prog. Phys. 27, 329 (1964).

[6] R.D. Gould, Thin Solid Films 125, 63 (1985).

[7] T.G. Abdel-Malik, A.M. Abdeen, H.M. El-Labany, A.A. Aly, Phys. Status Solidi A 72, 99 (1982).

[8] A.K. Hassan, R.D. Guld, Int. J. Electron. 69, 11 (1990).

[9] T.G. Abdel-Malik, Int. J. Electron. 72, 409 (1992).

[10] F.W. Schmidlin, G.G. Roberts, Phys. Rev. B 0, 1578 (1974). 